\title{
Final Report—Next-Generation Solvers for Mixed-Integer Nonlinear Programs: Structure, Search, and Implementation
}

\section{Award Number/Names}

\author{
DOE AWARD Number: $\quad$ DE-FG02-08ER25861 \\ Recipient Organization: The Board of Regents of the UW System, 21 N. Park Street, Suite 6401, \\ Madison, WI, 53715-1218 \\ Project Title: Next-Generation Solvers for Mixed-Integer Nonlinear Programs: \\ Structure, Search, and Implementation \\ Principal InVESTIGators: Jeff Linderoth (PI), James Luedtke (co-PI), Andrew Miller (co-PI) \\ PRoJeCt DATES 8/15/2008-8/14/2012
}

This project was awarded jointly with a project from Argonne National Lab (PI: Sven Leyffer, co-PI, Todd Munson). This report focuses mainly on the contributions of UW-Madison researchers Linderoth, Luedtke, and Miller.

\section{Executive Summary}

The mathematical modeling of systems often requires the use of both nonlinear and discrete components. Problems involving both discrete and nonlinear components are known as mixed-integer nonlinear programs (MINLPs) and are among the most challenging computational optimization problems. This research project added to the understanding of this area by making a number of fundamental advances. First, the work demonstrated many novel, strong, tractable relaxations designed to deal with non-convexities arising in mathematical formulation. Second, the research implemented the ideas in software that is available to the public. Finally, the work demonstrated the importance of these ideas on practical applications and disseminated the work through scholarly journals, survey publications, and conference presentations.

\section{Comparison of Goals/Objectives}

The primary goal of this work was to make strides in the practical ability of software to be able to solve optimization problems involving both nonlinear and discrete components. In pursuing this goal, we aimed to correct the mismatch between natural optimization models and available robust optimization software. Our work under this research project has made significant progress towards achiving this goal. In Table 1 is the original proposed work plan, along with the initials of the PIs originally tasked with leading developments in each area. The work turned out to be much more collaborative than expressed in the table, with PIs making significant contributions on areas not originally assigned as their primary responsibility. Further, as is often they case in exploratory research, the exact direction of the work is being driven by the discoveries made. In Section 4, our progress on these areas are described in more detail, as well as advances made in ths areas of computations and software. 
Table 1: Project Timeline, Milestones and Responsible Personnel by Year and Topic.

\begin{tabular}{llll} 
& Year 1 & Year 2 & Year 3 \\
\hline Structure & QP presolve [TM] & NLP presolve [TM] & MINLP presolve [TM] \\
& Trig-functions [SL] & Convex envelopes [AM] & Lifting \& MIR [AM] \\
& Disjunctive cuts [JRL] & Extended formulation [JRL] & \\
\hline Search & Strong branching [JTL] & MINLP heuristics [JTL] & Macro-partition [AM] \\
\hline Implementation & NLP restarts [SL] & Nonconvex [SL] & Parallel solver [JTL] \\
\hline
\end{tabular}

\section{Summary of Project Activities}

In this multi-year project, we considered

- Analytical descriptions of the convex hull of a bounded bilinear term;

- Characterization and approximability results for multilinear sets;

- Approximate extended formulations for the convex hull of multilinear sets, and the implementation of these ideas in the software package MINOTAUR;

- Characterization of the convex hull of some practically important nonconvex sets;

- Demonstration of the effectiveness of the perspective reformulation for solving convex, separable MINLP instances.

- Extending the strength of the perspective reformulation to nonseparable quadratic forms with variable upper bounds. (Many of the new inequalities were obtained via lifting);

- Extensions of linearization-based algorithms to solve MINLPs that have a convex continuous relaxation which is not necessarily defined by convex functions; and

- Production of survey articles describing the modern methods and challenges for solving MINLP.

In the following, we provide more details and citations for discoveries and contributions cenetered around these activities.

Convex Envelopes We have worked to characterize convex envelopes of nonconvex functions (or the convex hull of nonconvex sets). Our achievements in this area include the following:

1. Characterization of the convex hull of the set

$$
T \stackrel{\text { def }}{=}\left\{(x, y, z) \in \mathbb{R}^{3} \mid z=x y, \ell_{x} \leq x \leq u_{x}, \ell_{y} \leq y \leq u_{y}, \ell_{z} \leq z \leq u_{z}\right\} .
$$

In the case that $\ell_{z} \leq \ell_{x} \ell_{y}$ and $u_{z} \geq u_{x} u_{y}$, the convex hull is polyhedral, and given by the McCormick relaxation $\operatorname{conv}(T)=\mathcal{M}(T)$. Otherwise, the convex hull is not a polyhedron. This work is the first to derive an analytical expression for $\operatorname{conv}(T)[5]$. 
2. Investigation of the impact of convex envelope composition on the strength of the resulting convex relaxation. For a quadrilinear function $x_{1} x_{2} x_{3} x_{4}$, the two groupings $\left(\left(x_{1} x_{2}\right) x_{3}\right) x_{4}$ and $\left(x_{1} x_{2} x_{3}\right) x_{4}$ result in two different convex relaxations. In [3], we establish that the first composition results in a stronger relaxation and provide empirical evidence to that fact as well.

3. Characterization and approximability results for the convex hull of sums of multilinear terms. In particular, for the set

$$
X \stackrel{\text { def }}{=}\left\{(x, z) \in H \times \mathbb{R}^{m} \mid z_{i}=\phi_{i}(x) \forall i=1, \ldots m\right\},
$$

where $\phi_{i}(x)=\sum_{j \in J_{i}} a_{i j} \prod_{k \in K_{i j}} x_{k}$ is a multilinear function, and $H=\left\{x \in \mathbb{R}^{n} \mid \ell \leq x \leq u\right\}$. There is an exponentially-sized extended formulation for $\operatorname{conv}(X)$. The McCormick relaxation $\mathcal{M}(X)$ has the advantage of being polynomially-sized, but may be significantly weaker than the convex hull relaxation. The containment relationship $X \subseteq \operatorname{conv}(X) \subseteq \mathcal{M}(X)$ exists, but a fundamental question of importance to algorithm designers is to know the relative strength of the relaxations. In some special cases, we are able to establish analytic bounds on the ratio of the strength of the two relaxations, $\operatorname{conv}(X)$ and $\mathcal{M}(X)$. To our knowledge, these are the first approximation-ratio results for the strength of relaxations of global optimization problems. The results lend insight into the design of practical (non-exponentially sized) relaxations $\mathcal{R}(X)$ that are nearly as strong as $\operatorname{conv}(X)$ but which are closer in size to $\mathcal{M}(X)[19]$.

Extended Formulations Note that this work overlaps with the Convex Envelopes thrust of our research project. In computation, we plan to exploit aggregated extended formulations to achieve a combination of tightness and tractability. The primary computataional work to date was done as a proof of concept in the Ph.D. thesis of Namazifar [20]. The ideas have since been implemented in our open-source software package MINOTAUR, and a description of this work was given in the presentation [18].

Lifting Lifting a means through which strong inequalities for the convex hull of feasible solutions can be obtained. We considered the epigraph of a nonseparable quadratic function with variable upper bounds on the variables:

$$
Y=\left\{(x, z, v) \in \mathbb{R}_{+}^{n} \times \mathbb{B}^{n} \times \mathbb{R}_{+} \mid v \geq x^{T} Q x, x_{j} \leq z_{j} \forall j \in[n]\right\} \quad \text { for some matrix } Q \succeq 0 .
$$

The set $Y$ appears in applications such as portfolio management, data mining, and sparse optimization, so we are interested in obtaining a good approximation to $\operatorname{conv}(Y)$. Lifting was a key idea that helped us derive some new results for this important set.

1. We have shown that through a combination of variable transformation, restriction, convexification, and lifting, new, surprisingly-useful valid inequalities can be derived [14].

2. We demonstrate that valid inequalities for this set can be obtained by lifting inequalities for a related set without binary variables (QPB), introduced in [8]. In [10], we close a theoretical gap in the knowledge about the set (QPB), and we characterize the strength of different classes of lifted QPB inequalities. We show that one class, lifted-posdiag-QPB inequalities, capture no new information from the binary indicators. However, we demonstrate the importance of the other class, called 
lifted-concave-QPB inequalities. We show that all lifted-concave-QPB inequalities define the relevant convex hull for the case of convex quadratic programming with indicator variables. Second, we show that perspective inequalities are a special case of lifted-concave-QPB inequalities, and we further show that adding the perspective constraints to a semidefinite programming relaxation of convex quadratic programs with binary indicators results in a problem whose bound is equivalent to the recent optimal diagonal splitting approach of Zheng et al. [23]. Finally, we show the separation problem for liftedconcave-QPB inequalities is tractable if the number of binary variables involved in the inequality is small.

MINLP Presolve/Strong Relaxations In [11], we studied mixed integer nonlinear programs (MINLP)s that are driven by a collection of indicator variables where each indicator variable controls a subset of the decision variables. An indicator variable, when it is "turned off", forces some of the decision variables to assume fixed values, and, when it is "turned on", forces them to belong to a convex set. Many practical MINLPs contain integer variables of this type. We give an explicit characterization of the convex hull of the union of a point and a bounded convex set defined by analytic functions. This gives rise to a novel strong reformulation technique that we called the perspective reformulation. Further, we show that for many classes of problems, the perspective reformulation can be expressed via conic quadratic constraints, and thus relaxations can be solved via second-order cone programming.

Bound tightening is an important component of algorithms for solving nonconvex Mixed Integer Nonlinear Programs. A probing algorithm is a bound-tightening procedure that explores the consequences of restricting a variable to a subinterval with the goal of tightening its bounds. In [21], we propose a variant of probing where exploration is based on iteratively applying a truncated Branch-and-Bound algorithm. As this approach is computationally expensive, we use a machine-learning based classifiers to infer whether or not the probing algorithm should be employed.

New Linearization-based Algorithms Existing linearization-based algorithms assume the constraints of a MINLP are of the form $g_{j}(x) \leq 0$, where $g_{j}$ is a convex function. This prevents their application to problems in which the convex relaxation is convex, but the functions $g_{j}(x)$ defining the constraints are not convex. This situation occurs, for example, if $g_{j}(x)$ is a quasiconvex function. In [13], we derive two linearization-based algorithms that can solve such problems to optimality. These algorithms are called linearization-based because they rely on outer approximating linear programming relaxations constructed by taking linearizations of the nonlinear functions. On many classes of instances, such algorithms perform better than nonlinear-programming based branch-and-bound, which requires solving a nonlinear program at every node in the search tree. We find that these proposed algorithms are competitive with linearization-based algorithms that explicitly assume the functions $g_{j}(x)$ in the constraints are convex. Thus, these algorithms allow a wider range of problem classes to be solved without sacrificing computational performance.

Computations/Software In [1], we descibe FilMINT, a new solver for convex mixed integer nonlinear programs (MINLPs) that implements a linearization-based algorithm. The solver is based on an algorithm of Quesada and Grossmann that avoids the complete re-solution of a master mixed integer linear program (MILP) by adding new linearizations at open nodes of the branch-and-bound tree whenever an integer solution is found. We employ cutting planes, primal heuristics, and other well-known MILP enhancements 
for MINLPs, and we present detailed computational experiments that show the benefit of such advanced MILP techniques. We offer new suggestions for generating and managing linearizations that are shown to be efficient on a wide range of MINLPs. By carefully incorporating and tuning all these enhancements, an effective solver for convex MINLPs is constructed.

Application: Product Pooling The pooling problem consists of finding the optimal quantity of final products to obtain by blending different compositions of raw materials in pools. Bilinear terms are required to model the quality of products in the pools, making the pooling problem a non-convex continuous optimization problem. We studied an important generalization of the pooling problem where a fixed cost is imposed for using a raw material in apool. In [9], we derived four classes of strong valid inequalities for the problem and demonstrate that the inequalities dominate classic flow cover inequalities. We showed how the inequalities could be separated in polynomial time, and we gave computational results to demonstrate their utility.

Application: Engineering Design Optimal design applications are often modeled by using categorical variables to express discrete design decisions, such as material types. A disadvantage of using categorical variables is the lack of continuous relaxations, which precludes the use of integer programming techniques. In [2], we show how to express categorical variables with standard integer variable modeling techniques, and we illustrate this approach on a load-bearing thermal insulation system. Our new model allows us to employ black-box modeling languages and solvers and illustrates the interplay between integer and nonlinear modeling techniques. We present numerical experience that illustrates the advantage of the standard integer model.

Surveys Our work in the area of MINLP was partly notable for the number of survey articles that were invited or produced. In [6], we wrote a survey of recent progress and software for solving convex Mixed Integer Nonlinear Programs (MINLP)s. We demonstrated that by exploiting analogies to well-known techniques for solving Mixed Integer Linear Programs and incorporating these techniques into software, significant improvements have been made in the ability to solve this class of problems. The paper [7] was a survey srticle that described modern advances in disjunctive cutting planes for solving MINLPs. The perspective reformulation was surveyed in [12]. In addition, this work provided a computational study comparing various algorithmic techniques for solving perspective reformulations. The comprehensive survey [4] reviewed models, applications, and algorithms for both convex and non-convex MINLP.

\section{Product Produced}

\subsection{Publications}

The publications $[5,3,4,13,19,20,18,14,10,11,21,1,9,2,6,7,12]$ were all produced with support of from the grant award DE-FG02-08ER25861. 


\subsection{Presentations}

In addition to the publications described in the description of the completed research, the following presentations were made on research supported by DE-FG02-08ER25861 during the reporting period. Only the presenting author is listed.

- "Solving Mixed Integer Polynomial Optimization Problems with MINOTAUR," $21^{\text {st }}$ International Symposium on Mathematical Programming, Berlin, August 2012.

- “Great Minds Think Alike, Disjunctive Cuts for MINLP,” Valparaiso IP Workshop, Valparaiso, Chile, March 2012

- "Practical Polyhedral Relaxations for Multilinear Programs" $16{ }^{\text {th }}$ Combinatorial Optimization Workshop, Aussois, France, January, 2012.

- J. Luedtke, "Strong polyhedral relaxations for multilinear programs", 2012 INFORMS Optimization Society Conference, Miami, FL, February 2012.

- "Valid Inequalities \& Computations with Pooling Problems," INFORMS Annual Meeting, Charlotte, NC, November 2011.

- J. Luedtke "Strong polyhedral relaxations for multilinear programs," INFORMS Annual Meeting, Charlotte, NC, November 2011.

- J. Luedtke "Strong polyhedral relaxations for multilinear programs," 2011 DOE Applied Mathematics Program Meeting, Washington, D.C., October 2011.

- "Multi-term Relaxations for Multi-linear Programs," GERAD/Mprime Seminar, l’Université de Montréal, September 2011.

- J. Luedtke "Strong polyhedral relaxations for multilinear programs," SIAM Optimization Conference, Darmstadt, Germany, May 2011.

- "Computationally Effective Disjunctive Cuts for Convex Mixed Integer Nonlinear Programs," SIAM Optimization Conference, Darmstadt, Germany, May 2011.

- "Linear and Nonlinear Inequalities for a Nonseparable Quadratic Set," SIAM Optimization Conference, Darmstadt, Germany, May 2011.

- "Pooling Problems with Binary Variables," INFORMS Annual Meeting, Austin, TX, November 2010.

- J. Luedtke, "Relaxations of multilinear functions in mixed-integer nonlinear programming," INFORMS Annual Meeting, Austin, TX, November 2010.

- J. Luedtke, "Relaxations of multilinear functions in mixed-integer nonlinear programming," 2010 Mixed Integer Programming Workshop, Atlanta, GA, July, 2010.

- J. T. Linderoth, "Experiments with the Perspective Reformulation," OPENING LECTURE, European Workshop on Mixed Integer Nonlinear Programming, Marseille, France, April, 2010. 
- M. Kılınç, "Disjunctive Strong Branching Inequalities for Mixed Integer Nonlinear Programming," INFORMS Annual Meeting, San Diego, October 2009.

- J. T. Linderoth, "Strong Relaxations and Computations for Global Optimization Problems with Multilinear Terms," INFORMS Annual Meeting, San Diego, October 2009.

- M. Namizifar, "Convex Envelopes for Bounded Multilinear Functions," INFORMS 2009 Annual Meeting, San Diego, October 2009.

- M. Kılınç, "Inequalities from Strong Branching Information for Mixed Integer Nonlinear Programs," $20^{\text {th }}$ International Symposium on Mathematical Programming, Chicago, August 2009.

- J. T. Linderoth, "Strong Relaxations and Computations for Global Optimization Problems with Multilinear Terms," $20^{\text {th }}$ International Symposium on Mathematical Programming, Chicago, August 2009.

- J. T. Linderoth, "Feasibility Pump Heuristics for Mixed Integer Nonlinear Programs," $20^{\text {th }}$ International Symposium on Mathematical Programming, Chicago, August 2009.

- A. Miller, "Valid Inequalities, Separation, and Convex Hulls for Multilinear Functions," $20^{\text {th }}$ International Symposium on Mathematical Programming, Chicago, August 2009.

- J. T. Linderoth, "A Different Perspective on Perspective Cuts," Institut de Mathématiques de Bordeaux (IMB), Université Bordeaux 1, Bordeaux, France, July 2009

- M. Namizifar, "Linear Envelopes of Bounded Multilinear Functions", Bound Reduction Techniques for Constraint Programming and Mixed-Integer Nonlinear Programming Workshop, CPAIOR 2009, Pittsburgh, May 2009.

- J. T. Linderoth, "Inequalities from Strong Branching Information for Mixed Integer Nonlinear Programs," Computational Issues in Mixed Integer Nonlinear Programming Workshop, Institut de Mathematiques de Bordeaux (IMB), Université Bordeaux 1, March 2009.

- J. Luedtke, " Generating improved relaxations for global optimization with multilinear terms," Computational Issues in Mixed Integer Nonlinear Programming Workshop, Institut de Mathematiques de Bordeaux (IMB), Université Bordeaux 1, March 2009.

- J. Luedtke, “Generating improved relaxations for global optimization with multilinear terms," Optimization Applications in Engineering \& Applied Sciences (OptimA), University of Illinois at UrbanaChampaign, March 2009.

- J. T. Linderoth, "Latest Developments with FilMINT," INFORMS Computing Society National Meeting, Charleston, January 2009.

- J. T. Linderoth, "MINLP Wars: Building an Effective Solver for Convex Mixed Integer Nonlinear Programs," Institute for Mathematics and Its Applications, "Hot Topics" Workshop on Mixed Integer Nonlinear Programming, Minneapolis, November 2008.

- J. T. Linderoth, "A Different Perspective on Perspective Cuts," Argonne National Lab, Argonne, IL, October 2008. 


\section{Conclusions}

This report described the progress made on solving optimization problems whose difficulty arises from both nonlinear and discrete elements. A complete list of publications is given in the bibliography scetion. Support from the Department of Energy under grant agreement DE-FG02-08ER25861 is gratefully acknowleged.

\section{References}

[1] K. Abhishek, S. Leyffer, and J. T. Linderoth. FilMINT: An outer-approximation-based solver for nonlinear mixed integer programs. INFORMS Journal on Computing, 22:555-567, 2010.

[2] K. Abhishek, S. Leyffer, and J. T. Linderoth. Modeling without categorical variables: A mixed integer nonlinear program for the optimization of thermal insulation systems. Optimization and Engineering, 11:185-212, 2010.

[3] P. Belotti, S. Cafieri, J. Lee, L. Liberti, and A. J. Miller. On the composition of convex envelopes for quadrilinear terms. In A. Chinchuluun et al., editor, Optimization, Simulation, and Control, pages 1-16. Springer Verlag, 2013.

[4] P. Belotti, C. Kirches, S. Leyffer, J. Linderoth, J. Luedtke, and A. Mahajan. Mixed-integer nonlinear optimization. Acta Numerica, 2013. to appear.

[5] P. Belotti, A. J. Miller, and M. Namazifar. Linear inequalities for bounded products of variables. SIAG/OPT Views-and-News, 22(1):1-8, 2011.

[6] P. Bonami, M. Kılınç, and J. Linderoth. Algorithms and software for solving convex mixed integer nonlinear programs. In IMA Volumes in Mathematics and its Applications, volume 54, pages 1-40, 2012 .

[7] P. Bonami, J. T. Linderoth, and A. Lodi. Disjunctive cuts for mixed integer nonlinear programming problems. In Progress in Combinatorial Optimization, chapter 18, pages 521-541. ISTe-Wiley, 2011.

[8] S. Burer and A. Letchford. On nonconvex quadratic programming with box constriants. SIAM Journal on Optimization, 20(2):1073-1089, 2009.

[9] C. D'Ambrosio, J. T. Linderoth, and J. Luedtke. Valid inequalities for the pooling problem with binary variables. In Integer Programming and Combinatorial Optimization, 15th International IPCO Conference Proceedings, pages 117-129. Springer, 2011.

[10] H. Dong and J. Linderoth. On valid inequalities for quadratic programming with continuous variables and binary indicators. In IPCO 2013: The Sixteenth Conference on Integer Programming and Combinatorial Optimization, 2013. to appear.

[11] O. Günlük and J. Linderoth. Perspective relaxation of mixed integer nonlinear programs with indicator variables. Mathematical Programming Series B, 104:186-203, 2010. 
[12] O. Günlük and J. T. Linderoth. Perspective reformulation and applications. In IMA Volumes in Mathematics and its Applications, volume 154, pages 61-92, 2012.

[13] M. Hamzeei and J. Luedtke. Linearization-based algorithms for mixed-integer nonlinear programs with convex continuous relaxation. submitted for publication, 2013.

[14] H. Jeon and J. T. Linderoth. Experiments with the perspective reformulation. In P. Bonami, L. Liberti, A. Miller, and A. Sartenaer, editors, Proceedings of the European Workshop on Mixed Integer Nonlinear Programming, pages 27-30, April 2010.

[15] J. Linderoth, J. Luedtke, and M. Namazifar. Solving mixed integer polynomial optimization problems with MINOTAUR, August 2012. Presentation at the $21^{\text {st }}$ International Symposium on Mathematical Programming.

[16] J. Luedtke, M. Namazifar, and J. T. Linderoth. Some results on the strength of relaxations of multilinear functions. Mathematical Programming, Series B, 136:325-351, 2012.

[17] M. Namazifar. Strong Relaxations and Computations for Multilinear Programming. PhD thesis, Department of Industrial and Systems Engineering, University of Wisconsin-Madison, 2011.

[18] G. Nannicini, P. Belotti, J. Lee, J. Linderoth, F. Margot, and A. Wächter. A probing algorithm for MINLPs with early detection of failures by SVM. In CPAIOR 2011: The 8th International Conference on Integration of Artificial Intelligence and Operations Research, Lecture Notes in Computer Science, volume 6697, pages 154-169. Springer, 2011.

[19] X. Zheng, X. Sun, and D. Li. Improving the performance of MIQP solvers for quadratic programs with cardinality and minimum threshold constraints: A semidefinite program approach. Manuscript, 2010. 